\title{
Reviewer acknowledgement Biomed Tech volume 53 (2008)
}

The editors and the publishers wish to thank the following colleagues for their kind assistance in acting as referees for the journal this year:

Nicole Arweiler

Peter Augat

Rainer Bader

Christof Baltes

Günther Bauernfeind

Daniel Baumgartner

Detlef Behrend

Josip S. Bill

Wolfgang Birkfellner

Nick Bishop

Marc Bohner

Jens Bongartz

Martin Burghoff

André Butscher

Thorsten M. Buzug

Bernhard Clasbrummel

Richard Clayton

Silvia Comani

Gérard Crelier

Lutz Dürselen

Felix Eckstein

Michael Eiselt

Vilhelm Ekstrand

Roman Feil

Riccardo Fenici

Michael Fix

Renée Fuhrmann

José Manuel García Aznar

Beat Gasser

Frank Giessler

Birgit Glasmacher

Michael Hahn

Ulrich Hartmann

Joerg Andreas Hauser
Bernd Heinlein

Markus Heller

Bernd Henning

Karin Hensel

Martin Henri Hessmann

Jürgen Hochberger

Ulrich Hoffmann

Kai Holtappels

Matthias Honl

Natasa Honzikova

Jochen Jackowski

Oliver Jaekel

Marc Kachelrieß

Saim Kim

Klaus Peter Koch

Rüdger Kopp

Vladimir Krajca

Mirko Lippmann

Tony Lomax

Bartolomiej W. Loster

Hagen Malberg

Lorin P. Maletsky

Ivo Michiels

Michele Monno

Claudius Moor

Lars Morawietz

Urs Moser

Klaus D. Müller-Glaser

Ingo Nolte

Rudy Nuijts

Katia Parodi

Herfried Pessenhofer

Heidi-Lynn Ploeg

Mark Potse
Klaus Radermacher

Tammo Redeker

Heinz Redl

Helmut Sachs

Frank B. Sachse

Stefan Sauermann

Mark Scher

Alois Schloegl

Manfred Schmitt

Thomas Schmitz-Rode

Uwe Schneider

Bernd Schöller

Wolfgang Schreibmayer

Christine Schultze

Jess G. Snedeker

Wolf Ingo Steudel

Gudrun Stockmanns

Janette F. Strasburger

Olaf Such

Huw Summers

Peter Thomas

Roger Thull

Montserrat Vallverdu

Hans Van Oosterwyck

Jügen Wahrburg

Christian Wedemeyer

Daming Wei

Andre Weimann

Patrick Wolf

Heinz Wörn

Andreas Wree

Shaohua Xu 